\title{
The DOE Parallel Climate Model (PCM): The Computational Highway and Backroads
}

\author{
Thomas Bettge, Anthony Craig, Rodney James, \\ Vincent Wayland, and Gary Strand \\ National Center for Atmospheric Research, 1850 Table Mesa Drive, \\ Boulder, Colorado 80303 \\ (bettge, tcraig, rodney, wayland, strandwg) @ucar.edu
}

\begin{abstract}
The DOE Parallel Climate Model (PCM) is used to simulate the earth's climate system and has been used to study the climate of the $20^{\text {th }}$ century and to project possible climate changes into the $21^{\text {st }}$ century and beyond. It was designed for use on distributed memory, highly parallel, architectures. The computational requirements and design of the model are discussed, as well as its performance and scalability characteristics. A method for port validation is demonstrated. The shortcomings of the current model are summarized and future design plans are presented.
\end{abstract}

\section{Introduction}

The U.S. Department of Energy (DOE) Parallel Climate Model (PCM), constructed at the National Center for Atmospheric Research (NCAR) during the mid-1990s, is a comprehensive model of the earth's climate system, including fully active, scientifically contemporary, components of the atmosphere, ocean, land, river runoff, and sea ice.

The model has been used to simulate numerous climate states and climate change scenarios, including control climates of 1870, 1990, and 2060 (before and after the onset of anthropogenic activities), and the transient climate during 1870-2000 using observed greenhouse gas, sulfate aerosol, and solar variance forcing (Washington et al., 2000). Ensembles of future simulations extending to 2100 and beyond have been performed using the Intergovernmental Panel On Climate Change (IPCC) Third Assessment Report best case and worse case greenhouse gas forcing scenarios (Cubasch et al., 2001). In all, the PCM has been used to simulate over 7000 years of the earth's climate during the past four years.

At inception the target architectures of the PCM were massively parallel, distributed memory machines. The mid-1990s generation of Massively Parallel Processing (MPP) computer architectures forced the design of PCM into an all message passing application where the components themselves were stacked into the local memory on each computational node and run sequentially. Subsequently, as computer hardware 
at DOE facilities evolved with the deployment of Distributed Shared Memory (DSM) architectures and clusters of Symmetric Multi-Processors (SMPs), the PCM was ported and used successfully on a variety of these types of machines.

While the primary focus of the PCM is scientific investigation and discovery, computational performance is a high priority. The purpose of this paper is to review and present several computational aspects of the PCM including the early decision making process, the issue of portability across machines, and performance and productivity on available machines.

\section{Computational Requirements}

A major goal of the PCM was to use existing model components developed under the DOE Computer Hardware Advanced Mathematics Model Physics (CHAMMP) program. These individual models were targeted for machines with distributed memory across a large number of processors - the MPP class of architectures.

Many of the computational requirements of the PCM are obvious and include:

- must easily port across a number of platforms

- must validate across a number of platforms (i.e., must produce statistically identical climate simulations)

- must achieve a minimum level of high performance

- must use existing component models (PCM was not a model/software development project, but rather was charged to modify existing models to the extent necessary to achieve the requirements in a substantial way)

With respect to software development, the PCM was created using software engineering techniques which could focus on rapid development with customized software specific to, and documented among, a small group of developers. The limited scope of the project did not require the overhead and management which is recommended for larger projects which develop software for a much larger community.

\section{The Model Components}

The PCM is composed of individual models of the atmosphere, land, ocean, river runoff, and sea ice.

The atmospheric model is the parallel version of the NCAR Community Climate Model version 3 (CCM3) at T42 spectral truncation horizontal resolution (2.8 degrees latitude by longitude) with 18 vertical levels (see Kiehl et al., 1998). 
The land surface model is a one-dimensional model of the energy, momentum, and water exchanges between the earth's surface and the atmosphere, on the same horizontal grid as the atmosphere (Bonan, 1996). A river runoff scheme is included which directs freshwater from the land into the ocean according to predetermined (observed) basin flows (Branstetter et al., 2001).

The ocean component is the Los Alamos National Laboratory Parallel Ocean Program (POP) model with a displaced polar grid in the northern hemisphere. The grid has an average horizontal resolution of $2 / 3$ degress latitude and longitude, with 32 vertical levels (Dukowicz and Smith, 1994).

The sea-ice model provides a full dynamic and thermodynamic representation of the polar ice caps on a $27 \mathrm{~km}$ Cartesian grid centered over each pole (Washington et al., 2000).

Since the PCM is composed of components from independent model developers, considerable effort was required to create a software environment where the full PCM system would perform optimally, including the construction and implementation of a so-called coupler, which facilitates the exchange of information between individual components. The coupler steers the execution of the components and provides the mechanisms necessary to coordinate and support the transfer of data between the components, including computation of fluxes at the component interfaces, conservation of energy, and interpolation of data from one grid to another using the technique of Jones (1999).

\section{Computational Implementation}

When considering the implementation of the various components of PCM into a fully integrated computational model, it was necessary to evaluate the suitability of the available hardware to the problem at hand. Since the original target, the CrayT3D/T3E, did not have at the time an acceptable mechanism for allowing high speed message passing between active processes on separate partitions, it was decided to implement the PCM as a Single Program Multiple Data (SPMD) application, overlaying each component within the memory on the local compute node of the partition.

Fig. 1 shows a simplified representation of how the PCM is integrated sequentially in time. The primary time period over which the PCM components are synchronized, defined as the synchronous coupling interval, is one day. During this period the components each advance one day in sequence, with each component gathering or passing data as needed to the coupler for processing and distribution to the next component. All components and the coupler use all of the available computational nodes during the period in which they are active. Message passing within each component is achieved by use of the standard Message Passing Interface (MPI) library. 
Two major obstacles encountered in this scheme are (1) the horizontal grid is different for each component, and (2) the internal data decomposition employed in the distributed partitioning is different for each component. Thus, the partitioning strategy in the coupler became one of optimizing the use of MPI to gather and scatter information as needed when it is processed (in interpolating data from one grid to another, for example). Since the exchange of data between components involves very few floating point operations, the coupler activity is inherently non-scalable. For the PCM, this data movement problem was minimized by passing a majority of the data between components at the day boundaries.

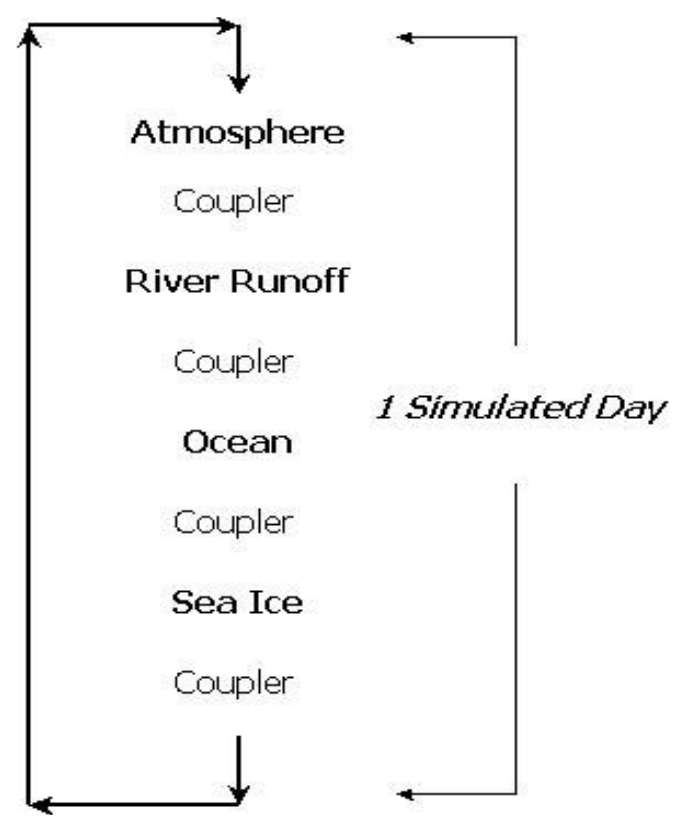

Fig. 1. Simplified integration sequence of the component models in PCM over the synchronous time interval of one day.

There are two characteristics of the current components used in PCM which limit its overall performance and scalability. First, the atmospheric model contains a onedimensional decomposition, which limits the number of processors that can be used in the solution to the number of latitude lines of the chosen horizontal grid resolution, which is 64. Second, the ocean model contains an elliptic equation solver which requires significant numbers of messages (MPI all-reduces) and many iterations during each two-hour timestep. When the problem is partitioned over more than 64 processors, the number of messages generated simply overwhelms the communication ability of the current target machines. 
Finally, the PCM is an all message passing application. It is partitioned in a way such that there is a straightforward, one-to-one, mapping of MPI processes to processors. There is no threading on compute nodes that contain multiple processors.

\section{Performance}

\subsection{Productivity}

The overall performance as well as productivity of the PCM on its targeted production machines is summarized in Fig. 2, which shows the number of simulated years per wallclock month achieved by a fixed version of PCM over the past several years. Climate modelers prefer to measure model performance in terms of scientific productivity rather than floating point operation counts for at least two reasons: (1) productivity, not floating point counts, is the goal of the scientific investigation, and (2) floating point operation counts are difficult to determine on many machines.

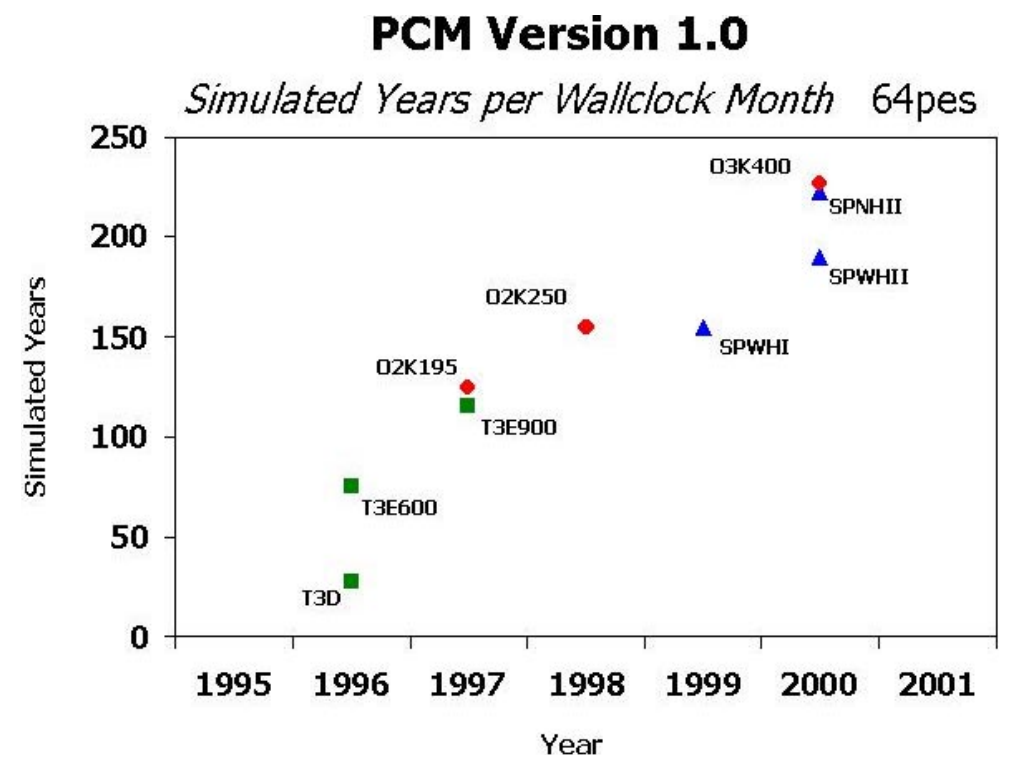

Fig. 2. PCM productivity for a fixed model version on 64pes of various machines from 1995 through 2000. Cray T3D and T3E, SGI Origin 2000 and 3000 (O2K, O3K, with chip clock speed given as last three digits), and IBM SP (WHI is WinterHawk I node, WHII is WinterHawk II node, and NHII is NightHawk II node) are shown. 
The original target machine of the PCM was the Cray-T3D, on which the rate of production was one simulated year per wallclock day using 64 processors. With the introduction of the Cray-T3E, especially the T3E-900, the production rate of $\mathrm{PCM}$ increased to the same range as other similar climate model codes which were designed for and used on parallel vector machines in production at various U.S. sites in 1997.

In successive years new machines became available. The design of PCM, in particular the use of a standard message passing library (MPI), allowed quick deployment of the model onto these machines. The PCM was given the enviable opportunity of being one of the first users at a number of sites, and the fact that we were able to take significant advantage of these opportunities is witness to the flexible design and portability of the PCM. The scientific returns with PCM for a fixed problem size increased rapidly as new, faster machines were introduced. Our current production rate for a single integration is six simulated years per wallclock day - a six-fold increase in productivity over a five-year period.

\subsection{Scalability}

The question arises as to how well PCM scales on these machines. Fig. 3 shows the model scaling for a variety of machines. Generally speaking, PCM scales reasonably well up to 32 processors and acceptably well up to 64 processors.

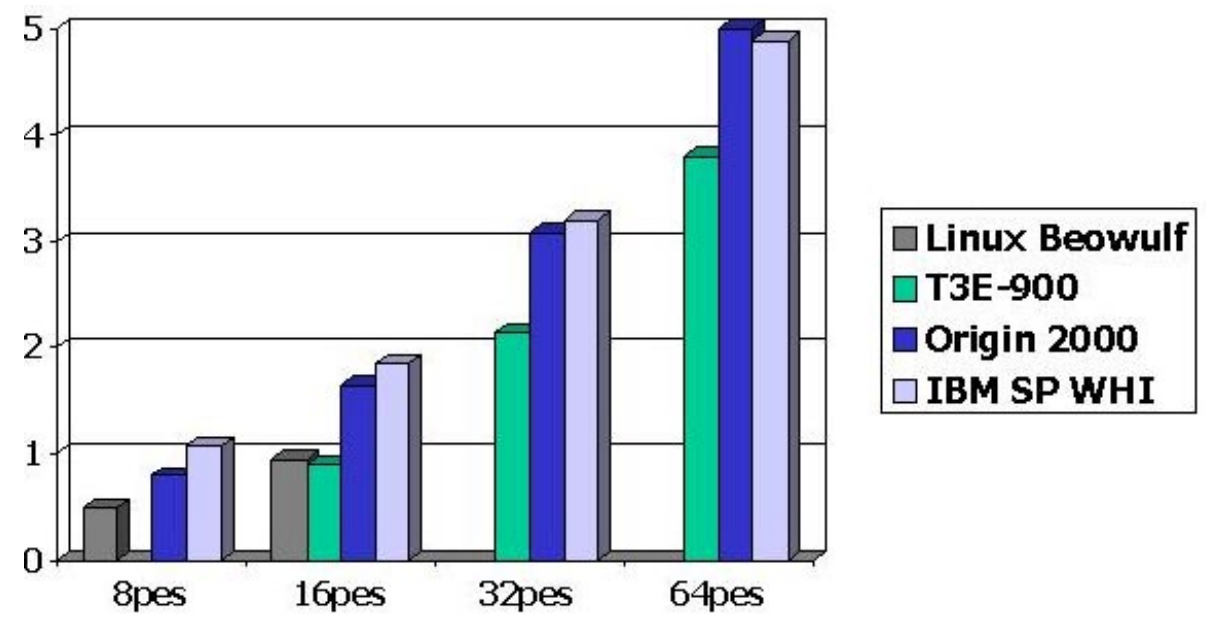

Fig. 3. PCM scalability from 8 to 64 processors on four machines. Simulated years per wallclock day. 
As was mentioned in section 4, the PCM is limited inherently to 64 processors due to the internal data decomposition of the atmospheric model. Additionally, the PCM limit of scalability is reached at 64 processors on the current generation of machines due to a combination of (1) the message latency and bandwidth on the hardware (an exception is the Cray-T3E), and (2) the message traffic generated by the model, in particular the elliptic equation solver in the ocean model. With respect to the communication within the components themselves, the individual model developers are exploring new methods to minimize messages, but no attempt has been made to address these issues within the PCM.

\section{Port Validation}

Use of the PCM across a variety of vendor platforms at a number of diverse computational centers involves validating the fact that the model was ported successfully to a new machine or site. The topic of port validation is important for obvious reasons. From platform to platform the model solutions, obtained using identical code and identical initial conditions, will diverge. How does one know the answers are correct?

The portability of PCM is validated by a procedure outlined by Rosinski and Williamson (1997). On a machine with which one has confidence in the solution, two simulations are produced. The second simulation is identical to the first, except that the solution path is forced to be different by introducing a random perturbation, which can be felt by the model at the precision of the machine, into the initial state. The growth of differences between the two solutions is considered to be representative of machine roundoff errors, and is assumed to accumulate only because of the initial, low-order perturbation. By design these errors are typical of the errors expected when the model is moved to a different architecture or when a new or different compiler is used, re-ordering some calculations. The difference of the simulation on the destination machine versus the original simulation on the first machine should exhibit similar characteristics as the differences generated by the two simulations on the first machine. Specifically, the differences during the first few timesteps should be close to the magnitude of machine rounding, and the differences should grow no faster than the differences from the two control simulations on the first machine.

Fig. 4 shows the result of a PCM port validation exercise. Note that the simulation differences, as measured by the RMS difference of surface temperature, between two machines are within one to two orders of machine rounding during the first few timesteps. During the first 144 timesteps (two days), the growth of the difference between the original and ported solutions do not exceed the growth of the initial perturbation introduced into the low-order bits of the original solution. In this case, the port is considered valid. 


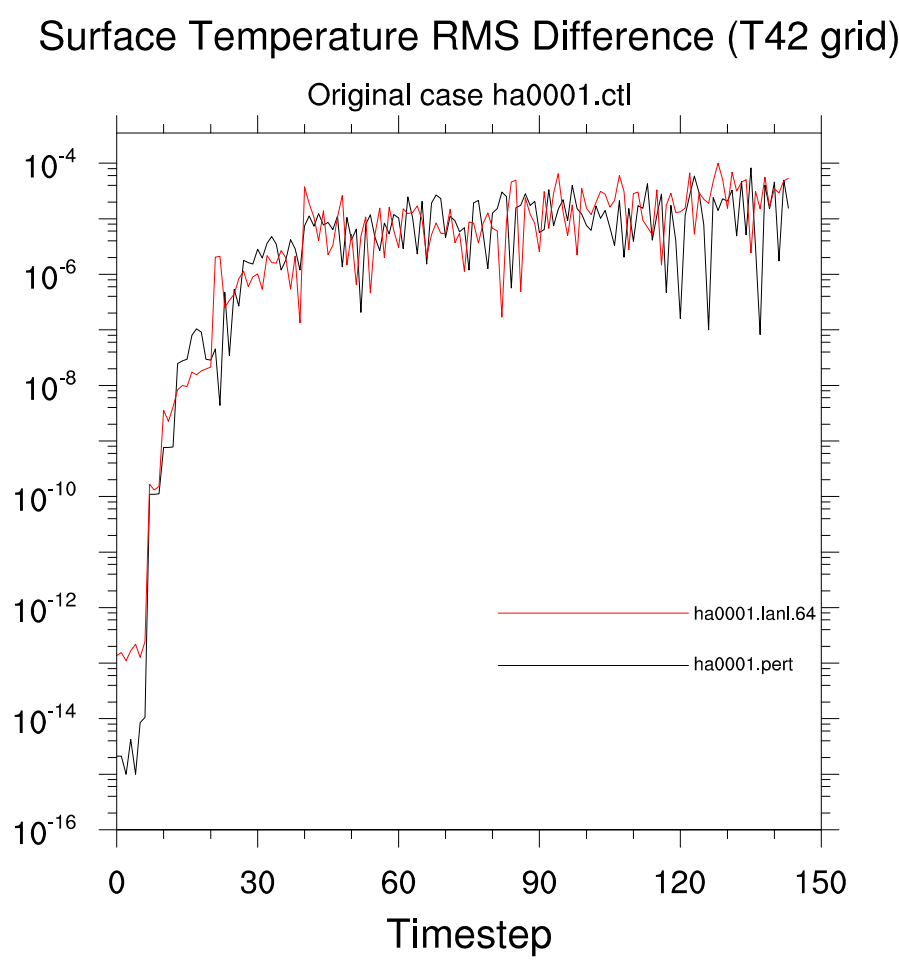

Fig. 4. Port validation of the PCM. The black line is the difference between two simulations on the control machine, which differ only because of an initial, low-order perturbation. The red line is the difference between two identical simulations produced on different architectures.

With climate modeling, it is equally important that the original and ported climate states are statistically identical. This can be accomplished only by making longer integrations and comparing the climates of the two states. This test is also part of the standard PCM validation procedure.

\section{Scientific Results}

The overall ability of PCM to simulate the earth's climate is demonstrated by the model's ability to simulate the climate during recent past, shown in Fig. 5. Arguably, the model reproduces the observed changes during the past 130 years in the globally averaged surface temperature. This version of the model was forced using observed greenhouse gas values (which act to produce heating at the surface), observed atmospheric sulfate aerosols (which act to produce cooling at the surface), and observed values of the incoming solar energy at the top of the atmosphere (which is observed to have slight variations over time). It is believed that the observed 
warming at the surface of the earth is caused by the net effect of changes in the radiative forcing on the earth, of which these three are major contributors.

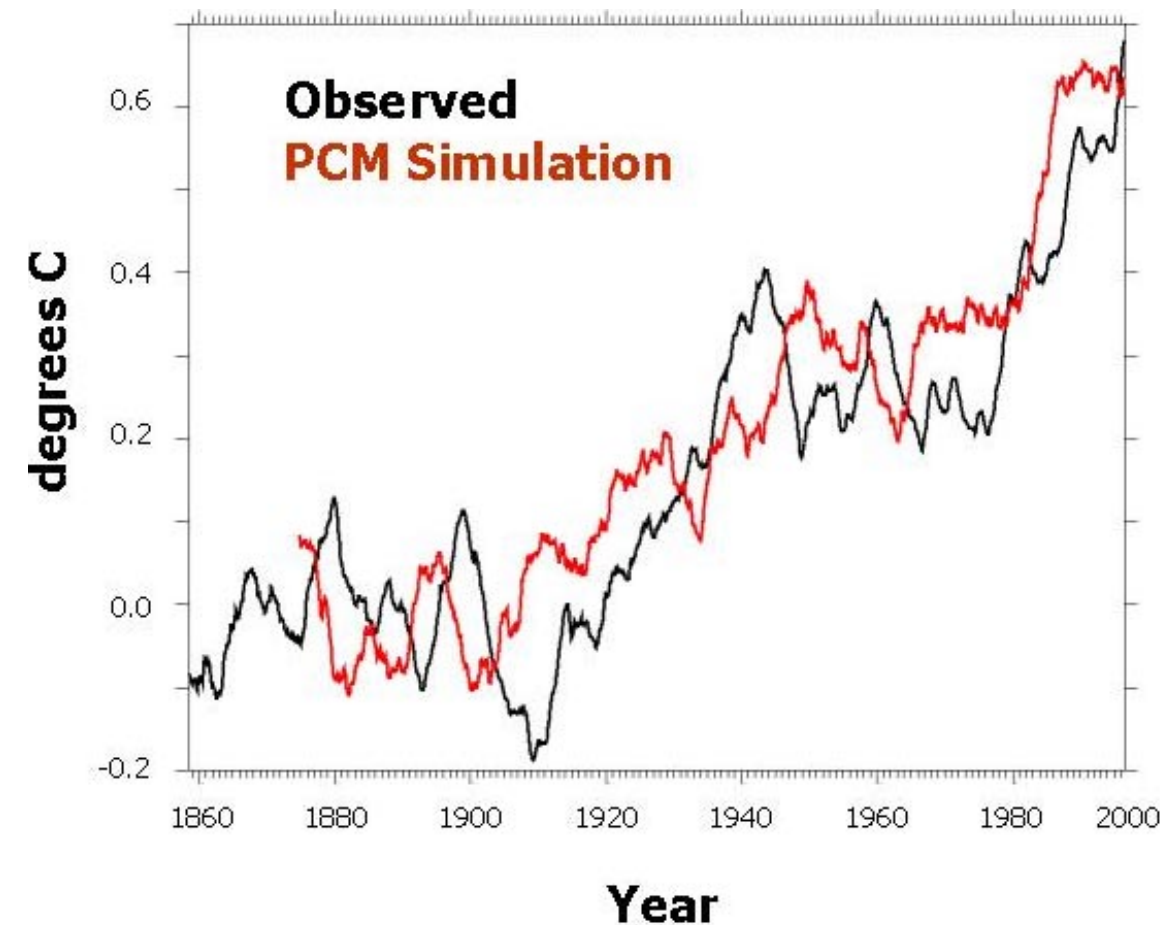

Fig. 5. Global surface temperature anomaly in degrees centigrade for 1860-1999. Observed is the black line. PCM simulation is the red line.

The magnitude of the temperature change, as well as the specific causes responsible for the change, are the subject of continuing scientific debate. In addition, the coarse resolution of the horizontal and vertical grid at the earth's surface is insufficient to allow definitive conclusions to be drawn concerning potential regional climate changes. The answers to questions like these lie, at least partially, in the effectiveness with which scientists can obtain and use increased computational power.

\section{Future}

At NCAR a climate model similar to the PCM was build with funding from the National Science Foundation - the Climate System Model (CSM) (CSM Model Plan, 2000). The CSM was designed originally and primarily for shared memory, vector parallel, architectures. Over the next year the PCM and the CSM will combine 
efforts, and a new model is currently under construction and will contain the following features, which address many of the original PCM shortcomings:

- individual components will integrate in Multiple Instruction Multiple Data (MIMD) mode; the components will run concurrently over the synchronous time period

- all components will contain a general two-dimensional data decomposition

- hybrid parallelism will be implemented within all components (threading on node, and message passing between compute nodes)

- communication between components via the coupler will be generalized and optimized

- $\quad$ specific problems (e.g., the elliptic equation solver in the ocean model) will be addressed with improved, computationally efficient, algorithms

The model should be ready for use in 2002 .

\section{References}

1. Bonan, G.B.: A Land Surface Model (LSM version1) for Ecological, Hydrological, and Atmospheric Studies: Technical Description and User's Guide. NCAR Technical Note NCAR/TN-417+STR. National Center for Atmospheric Research, Boulder, Colorado (1996)

2. Branstetter, M.L., Famiglietti, J.S., Washington, W.M., and Craig, A.P.: Using a 200-year simulation of a fully coupled climate system model to investigate the role of the continental runoff flux on the global climate system. Climate Variability, the Oceans, and Societal Impacts Symposium, American Meteorological Society, Albuquerque. (2001)

3. Community Climate System Model Plan (CCSM) for 2000-2005. Prepared by CCSM Scientific Steering Committee. National Center for Atmospheric Research, Boulder, Colorado (2000)

4. Cubasch, U., Meehl, G.A., Boer, G.J., Stouffer, R.J., Dix, M., Noda, A., Senior, C.A., Raper, S. and Yap, K.S.: Projections of Future Climate Change. Climate Change 2001: The Scientific Basis. In: Houghton, T, Ding Y., Griggs, D.J., Noguer, M. van der Linden, P., Dai, X., Maskell, K, and Johnson, C.I. (eds.) Contribution of Working Group I to the Third Assessment Report of the Intergovernmental Panel on Climate Change (IPCC). Cambridge University Press (2001)

5. Dukowicz, J.K.and Smith, R.D.: Implicit Free-Surface Method for the Bryan-Cox-Semtner Ocean Model. Journal of Geophysical Research (1994)

6. Jones, P.: First and Second -Order Conservative Remapping. Monthly Weather Review (1999)

7. Kiehl, J.T.: Simulation of the Tropical Pacific Warm Pool with the NCAR Climate System Model. Journal of Climate (1998) 11:1342-1355

8. Rosinski, J.M. and Williamson, D.L.: The Accumulation of Rounding Errors and Port Validation for Global Atmospheric Models. Journal of Scientific Computation, Vol. 18:2 (1997)

9. Washington, W.M., Weatherly, J.W., Meehl, G.A., Semtner, A.J., Bettge, T.W., Craig, A.P., Strand, W.G., Arblaster, J.M., Wayland, V.B., James, R., Zhang, Y.: Parallel Climate Model (PCM) Control and Transient simulations. Climate Dynamics, Vol. 16 (2000) 755-774 